\title{
Carotid artery intimal-medial thickness and left ventricular longitudinal systolic strain in patients with coronary artery disease
}

\begin{abstract}
Background: Carotid intima-media thickness (CIMT) is a non-invasive, inexpensive, reliable marker of coronary artery disease (CAD). LV global longitudinal strain function (LV GLS) can be accurately assessed by 2D speckle-tracking strain echocardiography (2D-STE).

Objectives: This study aimed to assess the relationship between carotid IMT and left ventricular global longitudinal strain (LV GLS) assessed by 2D speckle-tracking strain echocardiography (2D-STE) in patients with CAD. It also aimed to assess the ability of both LV global longitudinal strain (LV GLS) and carotid IMT to predict CAD severity.

Methods: It included 156 patients with suspected CAD by history of angina admitted electively for coronary angiography (CA). We excluded patients with acute coronary syndrome and concomitant disease or drug therapy which affect cardiac function. Patients underwent transthoracic echocardiography (TTE), 2-D speckle tracking echocardiography (2D-STE), B-mode ultrasound of the carotid arteries and coronary angiography. The patients were divided into two groups: group 1 (106 patients) with significant $(>70 \%)$ $\mathrm{CAD}$, and group 2 (50 patients) with non-significant $\mathrm{CAD}$ obstruction. Conventional 2D echocardiographic examinations were done by using General Electric VIVID 9 with M4S transducer, with a frequency of 1.5-4.3 MHz. Examinations included measurements of cardiac dimensions and LV ejection fraction. Images were obtained in the apical longaxis, four-chamber, and two chamber views for the global peak systolic longitudinal strain (PSLS). Measurement of CIMT was performed at the far wall of the common carotid artery (CCA) $1 \mathrm{~cm}$ proximal to the bifurcation.
\end{abstract}

Results: There were significant increase in the mean CIMT and significant decrease in GLS in group 1 compared to group $2(1.43 \pm 0.41$ vs $0.72 \pm 0.4, \mathrm{p}<0.001)$, and $(-12.2 \pm$ 2.93 vs $-16.65 \pm 3.5, \mathrm{p}<0.001)$ respectively. The cut-off value for LVGLS less than- $15.8 \%$ using ROC curve was a predictor of significant CAD with AUC 0.82, 95\%CI 0.71-0.94, $\mathrm{p}<$ 0.001 . The sensitivity, specificity and accuracy of GLS for detecting significant CAD were $96.2 \%, 68.0 \%$, and $87.2 \%$ respectively. The cut-off value of the CIMT $>1.1 \mathrm{~mm}$ using ROC curve was a predictor of significant CAD with AUC $0.89,95 \%$ CI $0.81-0.96, \mathrm{p}<0.001$. The sensitivity, specificity and accuracy of mean CIMT were $92.5 \%, 84.0 \%$ and $89.7 \%$. There was significant negative correlation between global LVGLS and mean carotid IMT $(r=$ $-0.88, \mathrm{p}<0.0001$ and positive correlation with $\mathrm{EF}(\mathrm{r}=0.37, \mathrm{p}=0.001)$.

Conclusion: Increased CIMT was associated with decreased LV function assessed by 2D strain echocardiography. Decreased GLS and increased CIMT were associated with significant CAD; So these findings support the use of CIMT measurements to predict subclinical LV dysfunction and the risk of CAD.

Keywords: carotid artery intimal-medial thickness, 1v global longitudinal strain function, speckle tracking echocardiography, $\mathrm{CAD}$
Volume 8 Issue I - 2017

Tamer Mostafa, Hanan Radwan

Department of Cardiovascular, Faculty of Medicine, Zagazig University, Egypt

Correspondence: Hanan Radwan, Department of Cardiovascular, Faculty of Medicine, Zagazig University, Egypt, Tel 201066381472, Email hananradwan67@yahoo.com

Received: December 07, 2016 | Published: January 09, 2017

\section{Introduction}

The atherosclerotic process is a generalized process affecting the arterial tree which begins in child-hood, progresses over decades and may remain clinically silent in the majority of people. In the coronary arteries, it remains asymptomatic until it progresses to cause a hemodynamically significant flow limiting lesion leading to symptoms of myocardial ischemia. However, a substantial proportion of patients progress abruptly from unapparent disease to a myocardial infarction or possible death, due to thrombus formation following acute rupture or erosion of non-stenotic plaques, ${ }^{1}$ Identification of patients at high risk in the early phase is critical to allow for modification of cardiovascular risk by effective preventive strategies. Coronary angiography (CA) is currently considered the gold standard for detecting obstructive coronary artery disease. However, its inability to detect early atherosclerotic changes, its invasive nature, as well as exposure to ionizing radiation, make it an unattractive primary screening tool in preventive cardiology. ${ }^{2}$

Ultrasound measurement of carotid intima-media thickness (CIMT) has become a valuable tool for detecting and monitoring progression of atherosclerosis. ${ }^{2}$ The American College of Cardiology Foundation/American Heart Association guidelines recommend CIMT measurements for patients at intermediate CVD risk. ${ }^{3}$ The longitudinally arranged subendocardial fibers are more vulnerable to ischemia due to their direct exposure to the intraventricular blood pressure and the anatomy of the coronary circulation. ${ }^{4-6}$ As a result, longitudinal function is impaired first in CAD. Measurements of 
longitudinal motion and deformation are therefore, the most sensitive markers of coronary artery disease especially in patients with severe coronary stenosis, where intermittent ischemia may result in subtle forms of stunning that may be detectable with strain measurements. Severe CAD is known to lead to LV dysfunction. However, the LV ejection fraction is usually normal at a relatively early stage ${ }^{7}$ which can be explained by that impairment in longitudinal systolic function is known to be compensated by augmentation of circumferential deformation. Global longitudinal peak systolic strain (GLS) can be detected by two-dimensional speckle tracking echocardiography (2D-STE). ${ }^{4-6}$

\section{Aim of the work}

This study aimed to assess the relationship between carotid IMT and left ventricular global longitudinal strain (LV GLS) assessed by 2D speckle-tracking strain echocardiography (2D-STE) in patients with coronary artery disease (CAD). It also aimed to assess the ability of both LV global longitudinal strain (LV GLS) and carotid IMT to predict CAD severity.

\section{Patients and methods}

\section{Patients}

The study was done in cardiology department in Zagazig University. It included 156 patients with suspected CAD by history of angina and either resting ECG changes or positive exercise stress test admitted electively for coronary angiography (CA). Exclusion criteria were; patients with acute coronary syndrome, previously known CAD as a history of myocardial infarction (MI), previous coronary artery bypass grafting surgery $(\mathrm{CABG})$ or percutaneous coronary intervention (PCI), patients with LV systolic dysfunction $(\mathrm{EF}<45 \%)$, patients with resting wall motion abnormalities, atrial fibrillation, left bundle-branch block, severe valvular disease, concomitant disease as connective tissue disease or drug therapy which affect cardiac function and patients with poor acoustic window unsuitable for speckle tracking echo. Patients underwent transthoracic echocardiography (TTE), 2-D speckle tracking echocardiography (2D-STE), B-mode ultrasound of the carotid arteries and coronary angiography. The patients were divided into two groups: group 1 (106 patients) with significant ( $>70 \%) \mathrm{CAD}$, and group 2 (50 patients) with non-significant $\mathrm{CAD}$ obstruction.

\section{Transthoracic echocardiographic (TTE)}

Examination was done by using General Electric VIVID 9 with M4S transducer, with a frequency of 1.5-4.3 MHz and high frame rate (60-90 frame/s), Examinations included measurements of cardiac dimensions and LV ejection fraction by M-mode method. ${ }^{8}$

\section{Strain analyses}

Two consecutive heart cycles at rest, from the three standards apical planes (four-chamber, two-chamber, and long-axis), were considered by conventional 2D gray scale echocardiography. In each of the apical views, the endocardial contour was manually drawn and tracking of deformation was automatically performed by the software, once visual confirmation of good quality tracking was given by the operator. The software algorithm automatically segmented the LV into six equidistant segments and selected suitable speckles in the myocardium for tracking. The software algorithm then tracked the speckle patterns on a frame by frame basis using the sum of absolute difference algorithm. Regional longitudinal peak systolic strain (RLS) was measured in all views between aortic valve opening and closing for the 6 basal, 6 midventricular, and 4 apical segments and 17th segment was apical cap and averaged from the 17 segments to provide global longitudinal peak systolic strain (GLS)8 (Figure 1A \& 1B).

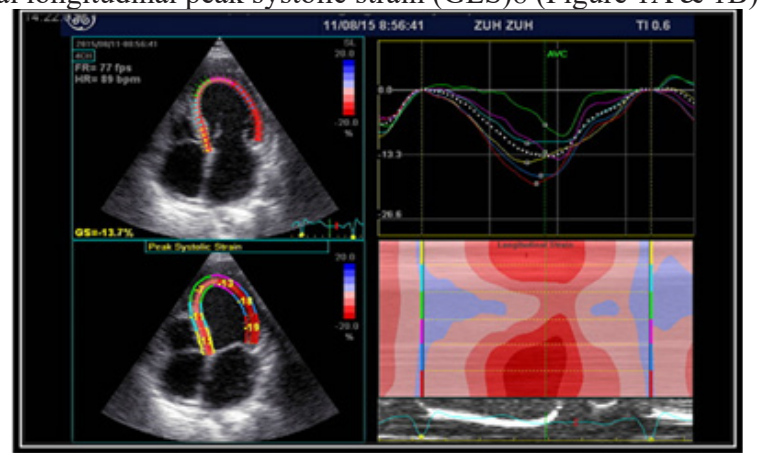

Figure IA Left panel demonstrates apical four-chamber view. Corresponding strain curves are shown in the right panel. GLS is decreased with strain values of - $13.7 \%$.

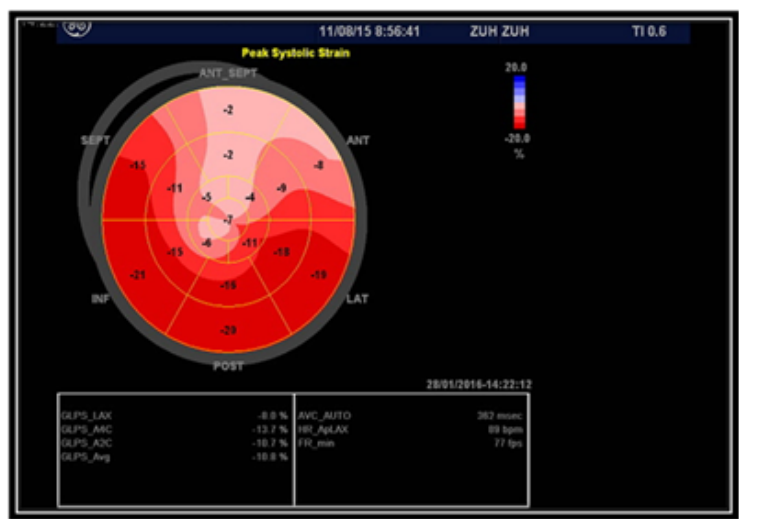

Figure IB Ball's eye showed averaged GLS $=-10.6 \%$.

\section{Carotid ultrasound}

The carotid ultrasound examination was carried out according to the American Society of Echocardiography guidelines. ${ }^{9}$ The extracranial common carotid arteries (CCAs) were sequentially assessed using a high-resolution 5- to $10-\mathrm{MHz}$ linear-array ultrasound transducer (GE-VingMed) by an experienced physician. Patients were examined in the supine position with the head angled approximately 45 degrees towards the contralateral side. IMT measurements were synchronized with the QRS complex on the ECG. Measurements were made in each carotid artery at the peak of the $\mathrm{R}$ wave where the lumen is widest. IMT was defined as the distance between the lumen-intima and media-adventitia borders of the vessel identified as a double line pattern in a longitudinal image. ${ }^{10}$ Measurement of CIMT was performed at the far wall of the common carotid artery (CCA) $1 \mathrm{~cm}$ proximal to the bifurcation over an area of $10 \mathrm{~mm}$ of the distal CCA recorded in longitudinal mode, ensuring the CCA was parallel to the transducer surface (horizontal in the image). Focal zone was set to the far CCA wall, and gain was adjusted high enough so echoes were just observed in the lumen. The reader selected frames with good perpendicular alignment and image quality and adjusted IMT box position if necessary to ensure measurement of mean CIMT over the distal $10 \mathrm{~mm}$ of the far wall of the CCA. For every patient, mean IMT measurements were taken at the same phase of the cardiac cycle (electrocardiography gated) on each artery (right/left). All CIMT measurements from both arteries were averaged to create mean CIMT. Plaque diameter was included in the CIMT measurements if present in the areas of interest.

Coronary Angiography (CA) was performed by the percutaneous femoral approach. Coronary angiograms were obtained for each 
coronary vessel in $\geq 2$ projections, and stenosis with $\geq 70 \%$ reduction of the arterial lumen area were considered significant. The analysis of the coronary angiograms was performed visually by an experienced operator who was blinded to the results of the echocardiographic examinations. ${ }^{11}$

\section{Ethics}

Informed parental consent was obtained to be eligible for enrollment into the study. The study was done according to the rules of the Local Ethics Committee of Faculty of Medicine, Zagazig University, Egypt.

\section{Statistical analysis}

All analyses were made using the "SPSS 17 for Windows" software package. Continuous variables were expressed as mean \pm standard deviation; categorical variables were expressed as percentages. Independent T-test was used to compare means, ANOVA to compare multiple groups. Chi-square test was used to compare percentages. Pearson's correlation coefficients were used to assess the strength of relationship between continuous variables. ROC curve was plotted to get cut off value, sensitivity and specificity. A $p$ value of less than 0.05 was considered significant.

\section{Results}

The patients were divided into two groups, group 1 (106 patients) with significant $\mathrm{CAD}>70 \%$ stenosis, and group 2 (50 patients) with non significant CAD. Comparison of the basic characteristic of the study population in patients with and without significant coronary obstruction are summarized in Table 1. Regarding age; it was 51.3 \pm 8.8 in group 1 versus $53.7 \pm 5.8$ in group $2, \mathrm{p}=0.28$. Sex and risk factors were comparable in both groups in spite high prevalence of risk factors in patients with significant CAD. Group 1 had significant decrease in GLS \& EF $(-12.2 \pm 2.93$ vs $-16.65 \pm 3.5, p<0.000)$, LVEF $(62.5 \pm 6.5$ vs $66.1 \pm 6.8, \mathrm{p}=0.033)$ and significant increase in mean CIMT $(1.43 \pm 0.41 \mathrm{vs} 0.72 \pm 0.4, \mathrm{p}<0.000)$ compared to group 2 .

Angiographic findings of the study population were summarized in Table 2: 50 patients (32.1\%) with non significant CAD-and 106 patients $(67.9 \%)$ with significant $\mathrm{CAD}+, 102$ patients $(96.2 \%)$ had LAD, 86 patients $(64.2 \%)$ had LCX, 38 patients $(35.8 \%)$ had RCA. 28 patients $(26.4 \%)$ had SVD, 32 patients $(30.2 \%)$ had DVD, 46 patients (43.4\%) had TVD. Diagnostic accuracy of CIMT \& GLS for detetection of significant epicardial coronary stenosis (Table 3, Figure $2 \& 3$ ); The optimal cutoff value of mean CIMT was $>1.1 \mathrm{~mm}$ AUC $0.89,95 \%$ CI $0.81-0.96, \mathrm{p}<0.000$ and for GLS was $<-15.8 \%$ AUC $0.82,95 \%$ CI $0.71-0.94 \mathrm{p}<0.000$. The sensitivity, specificity, positive predictive value, negative predictive value and accuracy of mean CIMT was $92.5 \%, 84.0 \%, 92.5 \% 84.0 \%$ and $89.7 \%$ respectively. The sensitivity, specificity, positive predictive value, negative predictive value and accuracy of mean GLS was $96.2 \%, 68.0 \%, 86.4 \%, 89.5 \%$ and $87.2 \%$ respectively.

Multivariate regression analysis for prediction severity of CAD showed that CIMT is the only predictor of CAD severity (OR 125, $95 \%$ CI $5.798-2694.9, p=0.002$ ) as shown in Table 4. For the group with normal global strain value, defined by the cut-off strain value of -15.8 , carotid IMT was $0.6 \pm 0.3 \mathrm{~mm}$ and for the group with reduced global strain values, carotid IMT was $1.4 \pm 0.4 \mathrm{~mm},(\mathrm{t}=8.5, \mathrm{p}<0.000)$. There were significant negative correlation between global LVGLS and mean carotid IMT $(\mathrm{r}=-0.88, \mathrm{p}<0.000)$ and positive correlation with EF ( $\mathrm{r}=0.37, \mathrm{p}=0.001)$ as shown in Table 5 \& Figure 4. GLS declined incrementally with increasing number of stenotic coronary arteries (GLS --16.65 $\pm 3.5,-14.1 \pm 2.01,-13.7 \pm 2.1 *$ and $-9.7 \pm$ $2.2 * * \$)$ for patients with non-significant CAD, SVD, DVD and TVD, respectively $\mathrm{p}<0.000$. CIMT increased incrementally with increasing number of stenotic coronary arteries (CIMT $0.72 \pm 0.3,1.15 \pm 0.38$, $1.4 \pm 0.3$ and $1.76 \pm 0.18$ ) for patients with non-significant CAD, SVD, DVD and TVD, respectively with $\mathrm{P}<0.000$ (Table 6).

Roc Curve

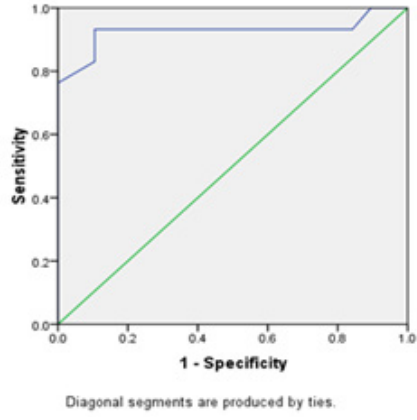

Figure 2 ROC curve for predicting significant CAD using GLS.

ROC Curve

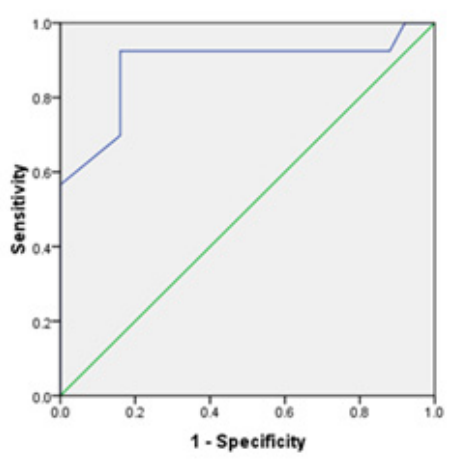

Diagonal segments are produced by ties.

Figure 3 ROC curve for predicting significant CAD using CIMT.

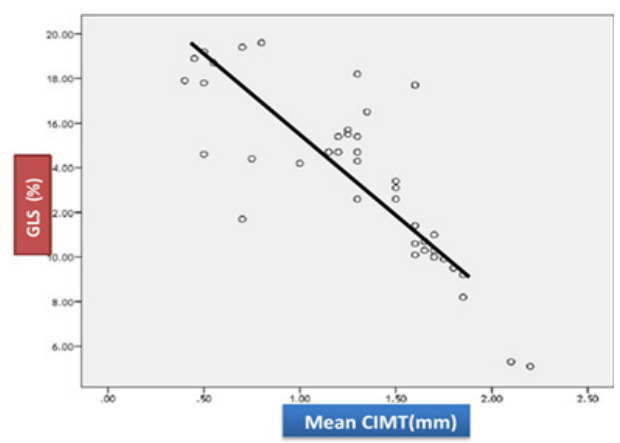

Figure 4 Negative correlation between GLS and mean CIMT.

\section{Discussion}

Impaired endocardial longitudinal function has been shown to be a sensitive marker for identifying early-stage diseased myocardium, particularly in patients with hypertrophy and ischemia, which may be exacerbated by elevated mechanical stress. ${ }^{12}$ Arterial remodeling, which means larger carotid IMT-to-lumen ratio, can result either wall thickening through collagen matrix deposition or increased internal diameter may share a similar pathophysiology with ventricular remodeling and worsened myocardial deformation, which may lead to the development of heart failure. ${ }^{13,14}$ 
Table I Comparison of the basic characteristic, echo and CIMT finding of the study population in patients with and without significant coronary obstruction

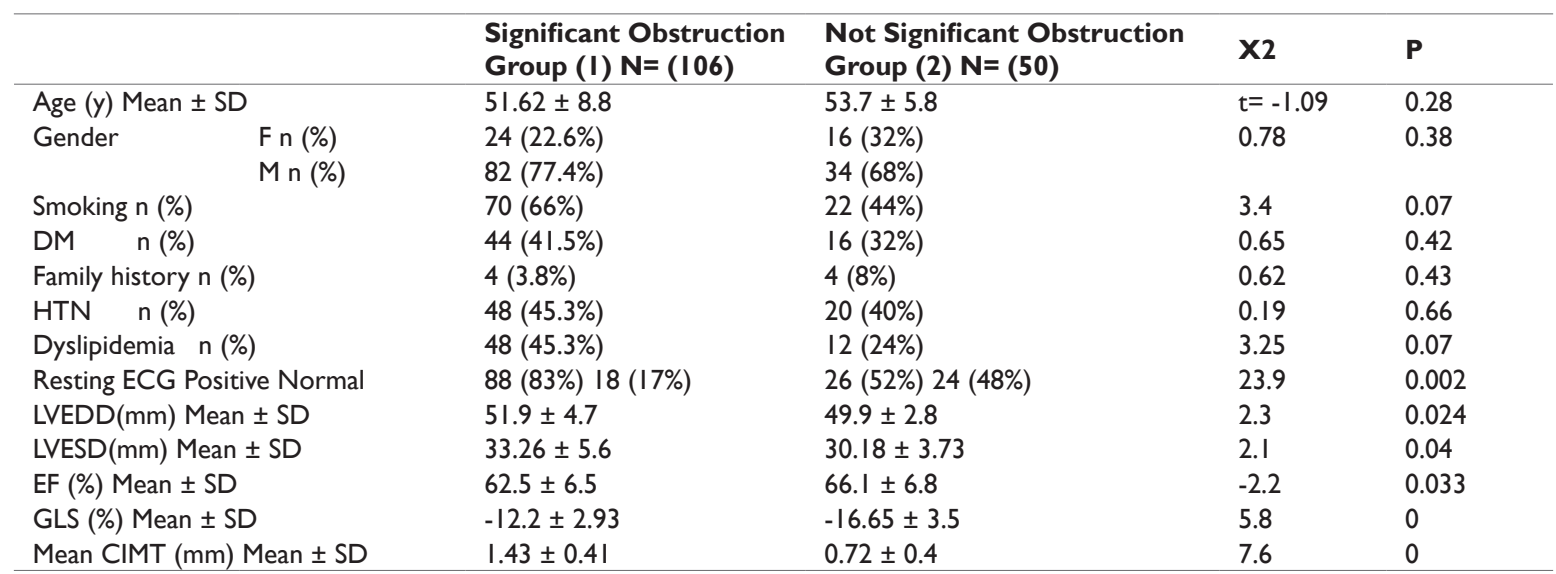

LV: Left Ventricle; EDD: End Diastolic Dimension; ESD: End Systolic Diamension; EF: Ejection Fraction; GLS: Global Longitudinal Strain; CIMT: Carotid Intimal Medial Thickness

Table 2 Angiographic findings of the study population

\begin{tabular}{ll}
\hline Angiographic Findings & N (\%) \\
\hline Non significant (group 2) & $50(32.1 \%)$ \\
Significant (group I) & $106(67.9 \%)$ \\
LAD & $102(96.2 \%)$ \\
LCX & $86(64.2 \%)$ \\
RCA & $38(35.8 \%)$ \\
SVD & $28(26.4 \%)$ \\
DVD & $32(30.2 \%)$ \\
TVD & $46(43.4 \%)$ \\
\hline
\end{tabular}

LAD: Left Anterior Descending; LCX: Left Circumflex, RCA: Right Coronary; SVD: Single Vessel Disease; DVD: Double Vessel Disease;TVD:Triple Vessel Disease.

Table 3 Diagnostic accuracy of CIMT \& GLS for detetection of significant epicardial coronary stenosis

\begin{tabular}{|c|c|c|c|c|c|c|c|c|}
\hline & AUC & $95 \% \mathrm{Cl}$ & $\mathbf{P}$ & Sensitivity & Specificity & PVV & NPV & Accuracy \\
\hline CIMT $(\mathrm{mm})>\mathrm{I} . \mathrm{I}$ & 0.89 & $0.8 I-0.96$ & 0 & $92.50 \%$ & $84 \%$ & $92.50 \%$ & $84 \%$ & $89.70 \%$ \\
\hline GLS $(\%)<-15.8$ & 0.82 & $0.7 \mid-0.94$ & 0 & $96.20 \%$ & $68 \%$ & $86.40 \%$ & $89.50 \%$ & $87.20 \%$ \\
\hline
\end{tabular}

Table 4 Multivariate regression analysis for prediction severity of CAD

\begin{tabular}{llll}
\hline & OR & $\mathbf{9 5 \%} \mathbf{~ C l}$ & $\mathbf{P}$ \\
\hline LVEDD & 0.89 & $0.69-1.12$ & 0.38 \\
LVESD & 1.08 & $0.84-1.38$ & 0.56 \\
LVEF (\%) & 0.91 & $0.79-1.04$ & 0.18 \\
GLS (\%) & 0.85 & $0.58-1.3$ & 0.85 \\
Mean CIMT (mm) & 125 & $5.798-2694.9$ & 0.002 \\
\hline
\end{tabular}

Table 5 Correlation between GLS and other parameters CIMT and EF

\begin{tabular}{lll}
\hline & GLS (\%) & \\
\hline & R & P \\
\hline LVEDD (mm) & -0.287 & 0.012 \\
$\operatorname{LVESD~(mm)~}$ & -0.263 & 0.023 \\
Mean CIMT (mm) & -0.877 & 0 \\
EF (\%) & 0.369 & 0.001 \\
\hline
\end{tabular}

Table 6 Relation between severity of coronary angiographic findings and GLS and mean CIMT

\begin{tabular}{lllllll}
\hline & Not significant & SVD & DVD & TVD & F & P \\
\hline & N (50) & N (28) & N (32) & N (46) & & \\
\hline GLS (\%) & $-16.65 \pm 3.5$ & $-14.1 \pm 2.01$ & $-13.7 \pm 2.1 *$ & $-9.7 \pm 2.2^{* *} \$$ & 23.5 & 0 \\
Mean CIMT (mm) & $0.72 \pm 0.3$ & $1.15 \pm 0.38$ & $1.4 \pm 0.3 @$ & $1.76 \pm 0.18 * * *$ & 23.13 & 0 \\
\hline
\end{tabular}

*p: I.000 versus SVD (non sign.)

**\$p: 0.000 versus SVD\&DVD

@p: 0.029 versus SVD (sign.)

***p: 0.000 versus SVD\&DVD (highly sign.)

Citation: Mostafa T, Radwan H. Carotid artery intimal-medial thickness and left ventricular longitudinal systolic strain in patients with coronary artery disease. J Cardiol Curr Res. 20I7;8(I):I2-I8. DOI: I0.15406/jccr.20I7.08.00268 


\section{Association between GLS and severity of CAD}

We found that GLS was significantly lower among patients with significant $\mathrm{CAD}(>70 \%)$ than those with non-significant $\mathrm{CAD}$ $(<70 \%)$ with mean values of GLS were $(-12.2 \pm 2.93$ vs $-16.65 \pm$ $3.5, \mathrm{p}<0.000)$. This finding was similar to the results reported by previous studies. ${ }^{15-20} \mathrm{We}$ found that GLS less than $-15.8 \%$ may predict of significant obstructive CAD $(>70 \%)$ in $87.2 \%$ of patients with sensitivity $96.2 \%$ and specificity $68.0 \%$ as shown Table 3 , Figure 2. So we concluded that GLS measurements had high diagnostic accuracy in predicting significant CAD among patients presenting with stable chest pain. These findings were different from previous studies $15-20$ as they found that GLS measurements only have modest diagnostic accuracy in predicting obstructive CAD. This difference may be explained by lower cutoff value of GLS (-15.8 \%) in our study compared to other studies where their cut-off level of GLS varied between $-17.4 \%$ and $-19.7 \%$.

Shimoni et al. ${ }^{15}$ showed that GLS $\leq-19.7$ may predict significant obstruction $(>50 \%)$ of CAD with sensitivity $81 \%$ and specificity $67 \%$. Montgomery et al. ${ }^{16}$ found that GLS $\leq-17.8$ may predict significant obstruction $(>50 \%)$ of $\mathrm{CAD}$, with sensitivity $66 \%$ and specificity $76 \%$. Biering- Soerensen et al., ${ }^{17}$ showed that cut-off level of GLS was $\leq-18.4 \%$ for prediction of significant CAD $(>70 \%)$ with sensitivity $74 \%$ and specificity $58 \%$. Evensen et al. ${ }^{18}$ found the endocardial strain GLS value less than $-16.7 \%$ may predict significant obstruction ( $>50 \%$ ) of CAD with sensitivity $80 \%$ and specificity $60 \%$. Billehaug Norum et al., ${ }^{19}$ used 6 studies (778 patients with suspected CAD). They found the cut-off level of GLS for prediction of CAD+ varied between $-17.4 \%$ and $-19.7 \%$ with a sensitivity from $51 \%$ to $81 \%$ and specifcity between $58 \%$ to $81 \%$. So they concluded GLS measurements at rest only have modest diagnostic accuracy in predicting $\mathrm{CAD}+$ among patients presenting with chest pain. These finding possibly could be explained by the effect of diastolic function and afterload on GLS, and there were overlap in distribution of GLS values between the CAD+ and CAD- groups. Gaibazzi et al. ${ }^{20}$ found that GLS using vendor-independent software, has similar accuracy compared with stress-echo wall motion analysis for the prediction of significant CAD. They concluded that GLS $\leq-20.7$ may predict significant obstruction $(>50 \%)$ of $\mathrm{CAD}$, with sensitivity $81.6 \%$ and specificity $84.9 \%$

The optimal GLS diagnostic cutoff value varies significantly among previous studies could be explained by GLS may depend on the clinical characteristics of patients, the effect of diastolic function and their hemodynamic parameters (i.e. blood pressure) during image acquisition, ${ }^{21}$ by using different equipment, different design, vendordependent 2D-STE software and operator skills. ${ }^{16,22,23}$ Another data have indicated that GLS is more dependent on the 2D-STE software used, rather than ultrasound equipment used to acquire images; ${ }^{24-26}$ which makes the use of vendor-independent software particularly attractive to standardize GLS for widespread use. The possibility of using a fixed diagnostic GLS cutoff value to diagnose obstructive $\mathrm{CAD}$, independent of the ultrasound equipment used for images acquisition, would be required to increase the clinical usefulness of strain imaging. We found that GLS declined incrementally with increasing severity of CAD defined by increasing number of stenotic coronary vessels. This finding was similar to results of BieringSoerensen et al., ${ }^{17}$ and also the studies reported by Gaibazzi et al., ${ }^{20}$ and Choi et al., ${ }^{22}$ which showed similar GLS values through different CAD categories. Patients with normal coronaries had a mean GLS of $-22 \pm 1.5 \%$, single or double vessel disease $-19.4 \pm 2.4 \%$, and triplevessel or left main disease $-18 \pm 2.3 \%$.

\section{Correlation between CIMT and GLS}

Our study found that for the patients with normal global strain value, defined by the cut-off strain value of $\geq-15.8 \%$, the carotid IMT was $0.6 \pm 0.3 \mathrm{~mm}$ and for the patients with reduced GLS values $<-15.8 \%$, the carotid IMT value was $1.4 \pm 0.4 \mathrm{~mm}(\mathrm{p}<0.000)$. Also we found that GLS had highly significant negative correlation with mean CIMT $(p<0.000)$. Fernandes et al., ${ }^{27}$ support our result as their MESA study is a prospective observational study including four ethnic groups free from clinical cardiovascular disease using tagged MRI for assessement of LV function. They found increased carotid IMT was associated with alterations of myocardial strain parameters reflecting reduced systolic and diastolic myocardial function. These observations indicate a relationship between subclinical atherosclerosis and incipient myocardial dysfunction in a population free of clinical heart disease. Liao et al., ${ }^{28}$ demonstrated that enlarged carotid artery diameter was associated with reduction of myocardial deformation indices including global longitudinal, circumferential and radial strain in subjects with hypertension (HTN) and heart failure with preserved ejection fraction (HFpEF), so it could be a useful clinical marker in early identifying ventricular functional decline. Evensen et al. ${ }^{18}$ support our results as they found increased carotid IMT values were associated with decreased left ventricular function they stated that the group with normal endocardial strain, defined by the cut-off strain value of -16.7 , carotid IMT was $1.2 \mathrm{~mm} \pm 0.2$ and for the group with reduced strain, the carotid IMT was $1.7 \mathrm{~mm} \pm 0.5$. There was significant correlation between endocardial LVGLS and maximum carotid IMT $(p=0.006)$.

Therefore, the presence of increased carotid IMT may lead to detection of very early cardiac dysfunction which may benefit from cardiovascular risk management. B-mode ultrasound of the carotid artery is a non-invasive, inexpensive and widely used examination and may therefore be an additional appropriate tool for the assessment of possible CAD. IMT measurements in patients with cardiovascular risk factors may therefore be of value when estimating the risk of subclinical coronary artery disease.

\section{Correlation between GLS and EF}

We found that patients with significant $C A D$ had significant decrease of left ventricle ejection fraction ${ }^{62} .5 \pm 6.5$ vs $66.1 \pm 6.8$, p $=0.033$ compared to patients with non-significant CAD. This finding is in agreement with Sharma et al., ${ }^{29}$ The present study found that GLS had highly significant positive correlation with EF $(r=0.37, p=$ 0.001 ). This result in concordant with results of, Biering- Soerensen et al. ${ }^{17}$ Evensen et al., ${ }^{18}$ and Delgado et al., ${ }^{30}$

Delgado et al., ${ }^{30}$ showed a good correlation between GLS and biplane LVEF for the overall study population, 222 patients with CAD $(\mathrm{r}=0.83 ; \mathrm{p}<0.001)$. However, in patients with STEMI and patients with heart failure the correlation was less strong $(r=0.42$ and $r=0.62)$ respectively suggesting that these two parameters reflect different aspects of systolic LV function.

\section{Association between CIMT and severity of CAD}

The present study showed carotid artery IMT was higher in patients with significant CAD $+>70 \%(1.43 \pm 0.41$ vs $0.72 \pm 0.4$, $\mathrm{p}<0.000)$ compared to group 2 with non-significant CAD This finding was similar to the results reported by previous studies. ${ }^{18,31-33}$ Thickening of the carotid intima-media (CIMT) to just over $1.1 \mathrm{~mm}$ may prove to be predictive of significant CAD in nearly $89.7 \%$ of patients with sensitivity (92.5\%) and specificity (84.0\%). There was incremental significant increase in CIMT with increasing severity 
of CAD defined by increasing number of stenotic coronary vessels. Tarzamni et al., ${ }^{31}$ found that the mean CIMT was $(0.83 \pm 0.26)$ in significant CAD (stenosis $>70 \%$ ) compared to $(0.53 \pm 0.16)$ in non significant CAD $(\mathrm{p}=0.001)$. Holland et al., ${ }^{32}$ reported that a maximal CIMT value of $0.956 \mathrm{~mm}$ had $85.7 \%$ sensitivity and $85.1 \%$ specificity to predict angiographic CAD. Coskun et al., ${ }^{33}$ showed CIMT was higher in patients with significant CAD $(1.48 \pm 0.28 \mathrm{~mm}$ vs $0.78 \pm$ $0.21 \mathrm{~mm}$ ). CIMT thickening $>1.0$ was predictive of significant $(>$ $50 \%$ ) CAD. They found the mean CIMT was significantly higher in $\mathrm{CAD}+$ groups, with single vessel disease, multi-vessel disease, and left main CAD compared to CAD - group $(0.78 \mathrm{~mm})(1.2 \pm 0.34 \mathrm{~mm}$, $\mathrm{p}=0.02 ; 1.6 \pm 0.32 \mathrm{~mm}, \mathrm{p}=0.001 ;$ and $1.8 \pm 0.31 \mathrm{~mm}, \mathrm{p}=0.0001$, respectively.

\section{Limitations}

1) Small sample size 2) Since the study was cross-sectional, the clinical endpoints were not followed so prognostic value of GIS not determined 3) Potential selection bias due to the need to enroll only patients with normal resting wall motion 4) Segmental RLS was not assessed because there were different methods used for obtaining regional strain. as a 16 -segment or a 17 -segment anatomical model for LV so the results represent a problem as this anatomical model does not necessarily reflect the individual coronary artery distribution because there is individual variability in the coronary blood supply to myocardial segment, also we need for proper standardization of how to uniformly obtain regional longitudinal strain (RLS) data.

\section{Conclusion}

Increased carotid IMT values were associated with decreased left ventricular strain function. So the presence of increased IMT may lead to detection of very early cardiac dysfunction which may benefit from cardiovascular risk management. These findings support the use of carotid IMT measurements to predict the risk of coronary heart disease. In patients with suspected coronary artery disease, GLS assessed by $2 \mathrm{D}$-STE at rest can predict significant CAD. So, 2D-STE has the potential to improve the value of echocardiography in the detection of the $\mathrm{CAD}$, identifying high-risk patients and to provide more information for clinical physician. Increased CIMT is associated with the presence and extent of CAD. Patients with a mean CIMT over $1.1 \mathrm{~mm}$ have a high likelihood $(90 \%)$ of significant CAD. So ultrasound examination of the carotid arteries can effectively predict atherosclerosis of the coronary arteries.

\section{Acknowledgments}

None.

\section{Conflicts of interest}

Author declares there are no conflicts of interest.

\section{Funding}

None.

\section{References}

1. Glaser R, Selzer F, Faxon DP, , et al. Clinical progression of incidental, asymptomatic lesions discovered during culprit vessel coronary intervention .Circulation. 2005;111(2):143-149.

2. Nair SB, Malik R, Khattar RS. Carotid intima-media thickness: ultrasound measurement, prognostic value and role in clinical practice. Postgrad Med J. 2002;88(1046):69-699.
3. Greenland P, Alpert JS, Beller GA, et al. 2010 ACCF/AHA guideline for assessment of cardiovascular risk in asymptomatic adults. Executive Summary: a report of the American College of Cardiology Foundation/ American heart association Task Force on practice guidelines. Circulation. 2010;122(25):e584-e636.

4. Tsai WC, Liu YW, Huang YY, et al. Diagnostic value of segmental longitudinal strain by automated function imaging in coronary artery disease without left ventricular dysfunction. J Am Soc Echocardiogr. 2010;23(11):1183-1189.

5. Hoit BD. Strain and strain rate echocardiography and coronary artery disease. Circulation . 2011;4:179-190.

6. Jasaityte R, Heyde B, D'hooge J. Current state of three-dimensional myocardial strain estimation using echocardiography. $J$ Am Soc Echocardiogr. 2013;26(1):15-28.

7. Parato VM, Mehta A, Delfino D, et al. Resting echocardiography for the early detection of acute coronary syndromes in chest pain unit patients. Echocardiography. 2010;27(6):597-602.

8. Lang RM, Badano LP, Mor Avi V, et al. Recommendations for cardiac chamber quantification by echocardiography in adults: an update from the American Society of Echocardiography and the European Association of Cardiovascular Imaging. J Am Soc Echocardiogr. 2015;28(1):1-39.

9. Stein JH, Korcarz CE, Hurst RT, et al. Use of carotid ultrasound to identify subclinical vascular disease and evaluate cardiovascular disease risk: a consensus statement from the American Society of Echocardiography Carotid Intima-Media Thickness Task Force. Endorsed by the Society for Vascular Medicine. J Am Soc Echocardiogr. 2008;21(2):93-111.

10. Touboul PJ, Hennerici MG, Meairs S, et al. Mannheim carotid intimamedia thickness and plaque consensus (2004-2006-2011). An update on behalf of the advisory board of the 3rd, 4th and 5th watching the risk symposia, at the 13th, 15th and 20th European Stroke Conferences, Mannheim, Germany, 2004, Brussels, Belgium, 2006, and Hamburg, Germany, 2011. Cerebrovasc Dis. 2012;34(4):290-296.

11. Lincoff AM, Topol EJ. Illusion of reperfusion. Does anyone achieve optimal reperfusion during acute myocardial infarction? Circulation. 1993;88(3):1361-1374.

12. Rosen BD, Saad MF, Shea S, et al. Hypertension and smoking are associated with reduced regional left ventricular function in asymptomatic: individuals the Multi-Ethnic Study of Atherosclerosis. $J$ Am Coll Cardiol. 2006;47(6):1150-1158.

13. Fernandes VR, Polak JF, Cheng S, et al. Arterial stiffness is associated with regional ventricular systolic and diastolic dysfunction: theMultiEthnic Study of Atherosclerosis. Arterioscler Thromb Vasc Biol. 2008;28(1):194-201.

14. Pavlopoulos H, Nihoyannopoulos P. Pulse pressure/stroke volume: a surrogate index of arterial stiffness and the relation to segmental relaxation and longitudinal systolic deformation in hypertensive disease. Eur J Echocardiogr. 2009;10(4):519-526.

15. Shimoni S, Gendelmann G, Ayzenberg O, et al. Differential effects of coronary artery stenosis of myocardial function: The value of myocardial strain analysis for the detection of coronary artery disease. J Am Soc Echocardiogr. 2011;24(7):748-757.

16. Montgomery DE, Puthumana JJ, Fox JM, et al. Global longitudinal strain aids the detection of non-obstructive coronary artery disease in the resting echocardiogram. European Heart Journal Cardiovascular Imaging . 2012;13:579-587.

17. Biering Sørensen T, Hoffman S, Mogelvang R, et al. Myocardial strain analysis by 2-Dimensional speckle tracking echocardiography improves diagnostics of coronary artery stenosis in stable angina pectoris. Circ Cardiovasc Imaging. 2014;7:58-65. 
18. Evensen K, Sarvari SI, Rønning OM, et al. Carotid artery intimamedia thickness is closely related to impaired left ventricular function in patients with coronary artery disease: a single-centre, blinded, nonrandomized study. Cardiovasc Ultrasound. 2014;12:39.

19. Billehaug N, Vidar R, Edvardsen T, et al. Diagnostic accuracy of left ventricular longitudinal function by speckle tracking echocardiography to predict significant coronary artery stenosis. A systematic review. BMC Medical Imaging. 2015;15:25.

20. Gaibazzi N, Pigazzani F, Reverberi C, et al. Rest global longitudinal $2 \mathrm{D}$ strain to detect coronary artery disease in patients undergoing stress echocardiography: a comparison with wall-motion and coronary flow reserve responses. Echo Res Pract. 2014;1(2):61-70.

21. Burns AT, La Gerche A, D'hooge J, et al. Left ventricular strain and strain rate: characterization of the effect of load in human subjects. Ehjcimaging. 2009;20:283-289.

22. Choi JO, Cho SW, Song YB, et al. Longitudinal 2D strain at rest predicts the presence of left main and three vessel coronary artery disease in patients without regional wall motion abnormality. European Journal of Echocardiography. 2009;10(5):695-701.

23. Smedsrud MK, Sarvari S, Haugaa KH, et al. Duration of myocardial early systolic lengthening predicts the presence of significant coronary artery disease. J Am Coll Cardiol. 2012;60(12):1086-1093.

24. Risum N, Ali S, Olsen NT, et al. Variability of global left ventricular deformation analysis using vendor dependent and independent two-dimensional speckle-tracking software in adults. $\mathrm{J} \mathrm{Am} \mathrm{Soc} \mathrm{of}$ Echocardiogr. 2012;25(11):1195-1203.

25. Sun JP, Lee AP, Wu C, et al. Quantification of left ventricular regional myocardial function using two-dimensional speckle tracking echocardiography in healthy volunteers- a multi-center study. Int $J$ Cardiol. 2012;167(2):495-501.
26. Negishi K, Lucas S, Negishi $T$, et al. What is the primary source of discordance in strain measurement between vendors: imaging or analysis? Ultrasound in Med Biol . 2013;39(4):714-720.

27. Fernandes VR, Polak JF, Edvardsen T, et al. Subclinical atherosclerosis and incipient regional myocardial dysfunction in asymptomatic individuals: the Multi-Ethnic Study of Atherosclerosis (MESA). J Am Coll Cardiol. 2006;47(12):2420-2428.

28. Liao ZY, Peng MC, Yun $\mathrm{CH}$, et al. Relation of carotid artery diameter with cardiac geometry and mechanics in heart failure with preserved ejection fraction. J Am Heart Assoc. 2012;1(6):e003053.

29. Sharma M, Ganguly NK. Premature coronary artery disease in Indians and its associated risk factors. Vasc Health Risk Manag. 2008;1(3):217-225.

30. Delgado V, Sjoerd A, Claudia Y, et al. Relation between global left ventricular longitudinal strain assessed with novel automated function imaging and biplane left ventricular ejection fraction in patients with coronary artery disease. J Am Soc Echocardiogr. 2008; 21(11):1244-1250.

31. Tarzamni MK, Salehi R, Givian F, et al. Association of carotid intimamedia thickness with the presence and severity of coronary artery disease. Neurosciences (Riyadh). 2006;11(4):308-311.

32. Holland Z, Ntyintyane LM, Raal FJ, et al. Carotid intima-media thickness is a predictor of coronary artery disease in South African black patients. Cardiovascular Journal of Africa. 2009; 20(4):237-239.

33. Coskun U, Yildiz A, Esen OB, et al. Relationship between carotid intimamedia thickness and coronary angiographic findings: a prospective study. Cardiovasc Ultrasound. 2009;7:59. 